\title{
APPLICATIONS OF STIFFNESS-BASED EVALUATION METHOD TO ELEMENT IMPORTANCE OF TRUSS SYSTEMS
}

\author{
Jianguo CAI ${ }^{\mathrm{a}}$, b, Wenwen JIA, Jian FENG ${ }^{\mathrm{a}}$, b, Fang WANG ${ }^{\mathrm{a}}$, Yixiang $\mathrm{XU}^{\mathrm{c}}$ \\ ${ }^{a}$ Key Laboratory of C\& PC Structures of Ministry of Education, Southeast University, Nanjing, China \\ ${ }^{b}$ National Prestress Engineering Research Center, Southeast University, Nanjing, China \\ ${ }^{c}$ Department of Civil Engineering, StrathclydeUniversity, Glasgow, United Kingdom
}

Received 02 Nov 2015; accepted 30 Mar 2016

\begin{abstract}
Two structural performance indexes, making use of eigenvalues of stiffness matrix, are presented in the study for the evaluation of element importance in the progressive collapse analysis of space trusses. Both indexes are based on the consensus that the element transferring higher loads in the load path is generally more important in the structural system. The first index is formulated as change of the smallest stiffness after removal of specific element, and the second index is defined as determinant of the stiffness matrix. Several simple numerical examples are presented to investigate performance of the proposed indexes; and finally, a square pyramid space grid system is studied as an illustrative example.
\end{abstract}

Keywords: structural robustness, skeletal structures, element importance, stiffness, progressive collapse, truss system.

\section{Introduction}

Progressive collapse is not an emerging subject. Researchers have paid much attention to this issue since the Ronan Point apartment collapsed in 1968 (Ministry of Housing and Local Government 1968). Furthermore, some essential requirements for structural integrity and collapse resistance have to be satisfied in some practical structural design codes (Ellingwood 2005; Stylianidis, Nethercot 2015; Djamaluddin et al. 2015; Guo et al. 2015). To enhance structural robustness and integrity as primary design methods, the tie force method, alternate load path method, and specific local resistance method were proposed (DoD 2009; GSA 2003).

In the existing literature, frames have been extensively studied for their robustness and capacity in resistance of progressive collapse, while little attention has been paid to long-span systems. For example, Wang (1994) discussed the static response of space frames with progressive member failure, including the effects of geometric nonlinearity. However, the accidental collapses of the roof at Charles de Gaulle airport in France and Bart Lai Haer Skating Museum in Germany, both of which occurred in recent years, highlight the importance and urgency of research on progressive collapse of long-span space structures.

Usually, space trusses of the double-layer grid type are assumed to have sufficient redundancy because of their high degree of static indeterminacy. Therefore, re- moval of one member would cause force redistributions that can be accommodated by the remaining structure. However, Murtha-Smith showed that progressive collapse of space trusses could occur following the removal of one of critical members, when they are subjected to full service loading (Murtha-Smith 1988). Affan and Calladine's work also showed that the collapse criterion for a space truss is a function not only of its degree of static indeterminacy, but also of other structural parameters (Affan 1987; Affan, Calladine 1996). Hence, the criterion in terms of static indeterminacy is not scientifically robust in preventing progressive collapse of space trusses (Sebastian 2004).

Wu et al. (1993a, 1993b) stated that it is an effective way to study structural inherent weaknesses based on its structural form. The effect of snap-through behavior on the collapse mechanism of space trusses was investigated by Morris (1993). The dynamic response of truss-type structures, including the double layer grids, after the loss of member was also studied (Malla, Nalluri 1995). Blandford (1996) utilized constitutive relationships to model the elastic and inelastic member behavior during the progressive collapse of space truss structures. Sheidaii et al. (2001) presented a methodology based on energy methods to determine the dynamic snap-through response of trusses. A methodology was given by Malla et al. (2011) to incorporate the history dependent cyclic inelastic axial

Corresponding author: Jianguo Cai

E-mail: caijg_ren@hotmail.com 
deformation of trusses in the dynamics. In the analysis, both the external dynamic loading and the internal dynamic effects caused by member failure have been taken into consideration. Mashhadiali and Kheyroddin (2014) carried out the progressive collapse assessment of new hexagrid structural system for tall buildings. Milana et al. (2015) provided some insight on the residual strength of diagrid structures in the damaged state, which is modelled by the elimination of diagonal grids.

Malla and Serrette (1996) stated that initial lack of perfectness may cause prestressing in members. Particularly in the cases of double-layer grids with high degree of static indeterminacy, they may face unexpected failure at relatively low loads. Ei-Sheikh (1997) proposed a numerical method to study the sensitivity of space trusses subjected to both gradual and sudden member losses. Moreover, they studied the effect of composite action between a top concrete slab and a space truss on reducing this sensitivity. Nafday (2011) proposed a consequencebased structural design method for black swan events, which is accomplished through the conceptual development of a system capacity oriented design approach. In this method, structural members were designed for variable reliabilities based upon their contribution to causing adverse system consequences. The robustness of trusses was studied by both nonlinear static and dynamic analysis (Jiang, Chen 2012). The mechanism of internal force redistribution was investigated and the significance of the mechanism affecting the subsequent failure process after the initial removal of the member is revealed. Olmati et al. (2013) applied a methodology to a steel truss bridge that takes into account the consequences of extreme loads on the structure, focusing on the influence of the loss of primary elements on the structural load bearing capacity. Salem and Helmy (2014) analytically investigated the cause of the collapse of a truss bridge using the Applied Element Method. Kim and Park (2014) carried out the study on progressive collapse of special truss moment frames, which consist of steel columns and open-web truss girders rigidly connected to form effective seismic load-resisting systems. De Biagi (2016) studied the behavior of a metallic truss under progressive damage and defined a possible strategy to sustain damage subjected to random removal of one of its elements.

It should be noted that in most of these literatures, the initial failure member was chosen arbitrarily or by conceptual judgment. By contrast, in frame structures and shear bearing wall structures, the members that should be removed in the progressive analysis are specified in the codes (DoD 2009; GSA 2003). This motivates us to present two different structural performance indexes to evaluate the element importance of the space trusses in their progressive collapse analysis. Both structural performance indexes are incorporated into the same framework of element importance index, based on the consensus that elements transferring higher loads in the load path are generally more important in the structure: the first structural performance (or element importance) index is defined as (change in) structural stiffness after removing a element, where the tangent stiffness is chosen to represent the structural stiffness; in the second structural performance (or element importance) index, (change in) determinant of the stiffness matrix is evaluated.

\section{Structural stiffness of space trusses}

\subsection{Topology}

Suppose that a truss has $m$ members, $n$ free nodes and $n^{f}$ fixed nodes. Let $k$ denote a member, and let $i$ and $j$ $(i<j)$ denote two nodes. If member $k$ is connected to the nodes $i$ and $j$, then the $i^{\text {th }}$ and $j^{\text {th }}$ entries of the $k^{\text {th }}$ row of the connectivity matrix $\mathbf{C}^{S}\left(\in \mathrm{R}^{m \times\left(n+n^{f}\right)}\right)$ are 1 and -1 , respectively; otherwise, if nodes $i$ and $j$ are not connected by the member $k$, then the corresponding elements in $\mathbf{C}^{s}$ are zero. Hence, the connectivity matrix $\mathbf{C}^{s}$ can be defined as follows (Zhang, Ohsaki 2006):

$$
C_{(k, p)}^{s}=\left\{\begin{array}{cc}
1, & \text { for } p=i ; \\
-1, & \text { for } p=j ; \\
0, & \text { for other cases. }
\end{array}\right.
$$

For convenience, the fixed nodes are preceded by the free nodes in the numbering sequence. Thus, the connectivity matrix $\mathbf{C}^{s}$ can be partitioned into two separated parts as:

$$
\mathbf{C}^{s}=\left(\begin{array}{ll}
\mathbf{C} & \mathbf{C}^{f}
\end{array}\right)
$$

where $\mathbf{C}\left(\in \mathrm{R}^{m \times n}\right)$ and $\mathbf{C}^{f}\left(\in \mathrm{R}^{m \times n^{f}}\right)$ describe the connectivity of members connected by the free and the fixed nodes, respectively.

\subsection{Equilibrium analysis}

Let $\mathbf{x}, \mathbf{y}, \mathbf{z}\left(\in \mathrm{R}^{n}\right)$ and $\mathbf{x}^{f}, \mathbf{y}^{f}, \mathbf{z}^{f}\left(\in \mathrm{R}^{n^{f}}\right)$ denote the nodal coordinates of the free and fixed nodes in $x, y$ and $z$ directions, respectively. Let $e_{k}$ and $a_{k}$ denote the Young's modulus and cross-sectional area of member $k$, respectively. The length of member $k$ in the stressed and initially unstressed states are denoted by $l_{k}$ and $l_{k}{ }_{k}$, respectively. Assuming that truss bars (members) are made of linear elastic materials; i.e., the strain-stress relation is linear, its force density $q_{k}$ can then be written as follows when the strain is small (Zhang, Ohsaki 2006, 2015):

$$
q_{k}=\frac{1}{l_{k}}\left(e_{k} a_{k} \frac{l_{k}-l_{k}^{0}}{l_{k}^{0}}\right)=e_{k} a_{k}\left(\frac{1}{l_{k}^{0}}-\frac{1}{l_{k}}\right) .
$$

The force density vector $q\left(\in \mathrm{R}^{m}\right)$ is denoted by $\boldsymbol{q}=\left(q_{1}, q_{2}, \ldots, q_{m}\right)^{\mathrm{T}}$, where ()$^{\mathrm{T}}$ refers to transpose of the matrix or vector. Denote force density matrix $\mathbf{Q}\left(\in \mathrm{R}^{m \times m}\right)$ as the diagonal version of the force density vector $\boldsymbol{q} \cdot e_{k} a_{k}$ of member $k$ are the $(k, k)$ element of the diagonal matrix $\mathbf{K}^{*}\left(\in \mathrm{R}^{m \times m}\right)$. Hence, all the force densities can be given as: 


$$
\mathbf{Q}=\mathbf{K}^{*}\left(\mathbf{L}_{0}^{-1}-\mathbf{L}^{-1}\right)
$$

Subsequently, the equilibrium equations in each direction can be given as (Zhang, Ohsaki 2006, 2015; Ohsaki, Zhang 2006; Cai, Feng 2015):

$$
\begin{aligned}
& \mathbf{C}^{\mathrm{T}} \mathbf{Q C x}+\mathbf{C}^{\mathrm{T}} \mathbf{Q} \mathbf{C}^{f} \mathbf{x}^{f}=\mathbf{f}^{x}, \\
& \mathbf{C}^{\mathrm{T}} \mathbf{Q C y}+\mathbf{C}^{\mathrm{T}} \mathbf{Q} \mathbf{C}^{f} \mathbf{y}^{f}=\mathbf{f}^{y}, \\
& \mathbf{C}^{\mathrm{T}} \mathbf{Q C z}+\mathbf{C}^{\mathrm{T}} \mathbf{Q} \mathbf{C}^{f} \mathbf{z}^{f}=\mathbf{f}^{z},
\end{aligned}
$$

where $\mathbf{f}^{x}, \mathbf{f}^{y}, \mathbf{f}^{z}\left(\in \mathrm{R}^{n}\right)$ are the external nodal load vectors applied on the free nodes in $x, y$ and $z$ directions, respectively.

Denote $\mathbf{E}\left(\in \mathrm{R}^{n \times n}\right)$ and $\mathbf{E}^{f}\left(\in \mathrm{R}^{n \times n^{f}}\right)$ as:

$$
\begin{aligned}
\mathbf{E} & =\mathbf{C}^{\mathrm{T}} \mathbf{Q} \mathbf{C}, \\
\mathbf{E}^{f} & =\mathbf{C}^{\mathrm{T}} \mathbf{Q} \mathbf{C}^{f} .
\end{aligned}
$$

Then the equilibrium equations can be rewritten as:

$$
\begin{aligned}
& \mathbf{E x}+\mathbf{E}^{f} \mathbf{x}^{f}=\mathbf{f}^{x}, \\
& \mathbf{E} \mathbf{y}+\mathbf{E}^{f} \mathbf{y}^{f}=\mathbf{f}^{y}, \\
& \mathbf{E z}+\mathbf{E}^{f} \mathbf{z}^{f}=\mathbf{f}^{z} .
\end{aligned}
$$

\subsection{Stiffness matrices}

The tangent stiffness matrix of the truss is defined by partial differentiation of the external nodal load vector $\mathbf{f}=\left(\mathbf{f}^{x \mathrm{~T}}, \mathbf{f}^{y \mathrm{~T}}, \mathbf{f}^{z \mathrm{~T}}\right)$ with respect to nodal coordinate vector $\mathbf{X}=\left(\mathbf{x}^{\mathrm{T}}, \mathbf{y}^{\mathrm{T}}, \mathbf{z}^{\mathrm{T}}\right)$, which can be written as:

$$
\mathbf{K}=\frac{\partial \mathbf{F}}{\partial \mathbf{X}}=\left[\begin{array}{ccc}
\frac{\partial \mathbf{f}^{x}}{\partial \mathbf{x}} & \frac{\partial \mathbf{f}^{x}}{\partial \mathbf{y}} & \frac{\partial \mathbf{f}^{x}}{\partial \mathbf{z}} \\
\frac{\partial \mathbf{f}^{\mathrm{y}}}{\partial \mathbf{x}} & \frac{\partial \mathbf{f}^{y}}{\partial \mathbf{y}} & \frac{\partial \mathbf{f}^{y}}{\partial \mathbf{z}} \\
\frac{\partial \mathbf{f}^{z}}{\partial \mathbf{x}} & \frac{\partial \mathbf{f}^{z}}{\partial \mathbf{y}} & \frac{\partial \mathbf{f}^{z}}{\partial \mathbf{z}}
\end{array}\right],
$$

where $\mathbf{K}\left(\in \mathrm{R}^{3 n \times 3 n}\right)$ is known as the tangent stiffness matrix.

Partial differentiation of Eqn (7) with respect to $\mathbf{x}$ results in:

$$
\left\{\begin{array}{c}
\frac{\partial \mathbf{f}^{x}}{\partial \mathbf{x}}=\left(\frac{\partial \mathbf{E}}{\partial x_{1}} \mathbf{x}+\frac{\partial \mathbf{E}^{f}}{\partial x_{1}} \mathbf{x}^{f}, \frac{\partial \mathbf{E}}{\partial x_{2}} \mathbf{x}+\frac{\partial \mathbf{E}^{f}}{\partial x_{2}} \mathbf{x}^{f}, \ldots, \frac{\partial \mathbf{E}}{\partial x_{n}} \mathbf{x}+\frac{\partial \mathbf{E}^{f}}{\partial x_{n}} \mathbf{x}^{f}\right)+\mathbf{E}, \\
\frac{\partial \mathbf{f}^{\mathbf{y}}}{\partial \mathbf{x}}=\left(\frac{\partial \mathbf{E}}{\partial x_{1}} \mathbf{y}+\frac{\partial \mathbf{E}^{f}}{\partial x_{1}} \mathbf{y}^{f}, \frac{\partial \mathbf{E}}{\partial x_{2}} \mathbf{y}+\frac{\partial \mathbf{E}^{f}}{\partial x_{2}} \mathbf{y}^{f}, \ldots, \frac{\partial \mathbf{E}}{\partial x_{n}} \mathbf{y}+\frac{\partial \mathbf{E}^{f}}{\partial x_{n}} \mathbf{y}^{f}\right), \\
\frac{\partial \mathbf{f}^{z}}{\partial \mathbf{x}}=\left(\frac{\partial \mathbf{E}}{\partial x_{1}} \mathbf{z}+\frac{\partial \mathbf{E}^{f}}{\partial x_{1}} \mathbf{z}^{f}, \frac{\partial \mathbf{E}}{\partial x_{2}} \mathbf{z}+\frac{\partial \mathbf{E}^{f}}{\partial x_{2}} \mathbf{z}^{f}, \ldots, \frac{\partial \mathbf{E}}{\partial x_{n}} \mathbf{z}+\frac{\partial \mathbf{E}^{f}}{\partial x_{n}} \mathbf{z}^{f}\right),
\end{array}\right.
$$

where $x_{i}$ denotes the $x$-coordinate of the free node $i$. By using the definitions in Eqn (6), where $\mathbf{C}$ and $\mathbf{C}^{f}$ are constant matrices, we obtain:

$$
\left\{\begin{array}{c}
\frac{\partial \mathbf{E}}{\partial x_{i}}=\mathbf{C}^{\mathrm{T}} \frac{\partial \mathbf{Q}}{\partial x_{i}} \mathbf{C}, \\
\frac{\partial \mathbf{E}^{f}}{\partial x_{i}}=\mathbf{C}^{\mathrm{T}} \frac{\partial \mathbf{Q}}{\partial x_{i}} \mathbf{C}^{f} .
\end{array}\right.
$$

Because the member lengths $\mathbf{L}_{0}$ in the unstressed state is constant, the partial differentiation of $\mathbf{Q}$ with respect to the $x_{i}$ can be written as follows from Eqn (4):

$$
\frac{\partial \mathbf{Q}}{\partial x_{i}}=\mathbf{K}^{*}\left(\mathbf{L}^{-1}\right)^{2} \frac{\partial \mathbf{L}}{\partial x_{i}} .
$$

Moreover, $\mathbf{K}^{*}$ is constant, since the members are assumed to be linear elastic such that $e_{k}$ is constant and change in the cross-sectional area $a_{k}$ is negligible.

The coordinate differences $u_{k}, v_{k}$ and $w_{k}$ of the member $k$ connecting to nodes $i$ and $j(i<j)$ in $x, y$ and $z$ directions can be respectively calculated as follows:

$$
u_{k}=x_{i}-x_{j}, v_{k}=y_{i}-y_{j}, w_{k}=z_{i}-z_{j} .
$$

From the definition of the connectivity matrix, which has only two non-zero elements in its $k$-row, 1 and -1 corresponding to the nodes $i$ and $j$ connected by the member $k$, Eqn (12) can be rewritten as:

$$
\left\{\begin{array}{l}
u_{k}=\mathbf{C}_{k} \mathbf{x}+\mathbf{C}_{k}^{f} \mathbf{x}^{f}, \\
v_{k}=\mathbf{C}_{k} \mathbf{y}+\mathbf{C}_{k}^{f} \mathbf{y}^{f}, \\
w_{k}=\mathbf{C}_{k} \mathbf{z}+\mathbf{C}_{k}^{f} \mathbf{z}^{f},
\end{array}\right.
$$

where $\mathbf{C}_{k}$ and $\mathbf{C}_{k}{ }_{k}$ denote the $k$-th rows of $\mathbf{C}$ and $\mathbf{C}^{f}$, respectively. Hence, we can have the coordinate difference vectors $\mathbf{u}, \mathbf{v}$ and $\mathbf{w}\left(\in \mathrm{R}^{m}\right)$. In some cases, it might be more convenient for us to use the diagonal form of a vector, which is denoted by the capital letter. For example, $\mathbf{U}, \mathbf{V}$ and $\mathbf{W}$ are the diagonal forms of the coordinate difference vectors $\mathbf{u}, \mathbf{v}$ and $\mathbf{w}$, respectively.

Because the length $l_{k}$ of member $k$ has the following relation with its coordinate differences:

$$
l_{k}^{2}=u_{k}^{2}+v_{k}^{2}+w_{k}^{2},
$$

square of the length vector $\mathbf{l}^{2}\left(\in \mathrm{R}^{m}\right)$ can be written as follows using the coordinate difference vectors:

$$
\mathbf{l}_{k}^{2}=\mathbf{u}_{k}^{2}+\mathbf{v}_{k}^{2}+\mathbf{w}_{k}^{2} .
$$

The diagonal form of $\mathbf{I}$ is denoted by $\mathbf{L}\left(\in \mathrm{R}^{m \times m}\right)$.

From Eqn (15), partial differentiation of $\mathbf{L}$ with respect to $x_{i}$ leads to:

$$
\frac{\partial \mathbf{L}}{\partial x_{i}}=\mathbf{L}^{-1}\left(\mathbf{U} \frac{\partial \mathbf{U}}{\partial x_{i}}+\mathbf{V} \frac{\partial \mathbf{V}}{\partial x_{i}}+\mathbf{W} \frac{\partial \mathbf{W}}{\partial x_{i}}\right)
$$

From Eqn (13), we have:

$$
\frac{\partial \mathbf{U}}{\partial x_{i}}=\operatorname{diag}\left(\mathbf{C}_{i}\right), \frac{\partial \mathbf{V}}{\partial x_{i}}=0, \frac{\partial \mathbf{W}}{\partial x_{i}}=0 .
$$


Then we have:

$$
\begin{aligned}
\frac{\partial \mathbf{E}}{\partial x_{i}} \mathbf{x}+\frac{\partial \mathbf{E}^{f}}{\partial x_{i}} \mathbf{x}^{f} & =\mathbf{C}^{\mathrm{T}} \mathbf{K}^{*}\left(\mathbf{L}^{-1}\right)^{3} \mathbf{U} \operatorname{diag}\left(\mathbf{C}_{i}\right)\left(\mathbf{C} \mathbf{x}+\mathbf{C}^{f} \mathbf{x}^{f}\right) \\
& =\mathbf{C}^{\mathrm{T}} \mathbf{K}^{*}\left(\mathbf{L}^{-1}\right)^{3} \mathbf{U} \operatorname{diag}\left(\mathbf{C} \mathbf{x}+\mathbf{C}^{f} \mathbf{x}^{f}\right) \mathbf{C}_{i} \\
& =\mathbf{C}^{\mathrm{T}} \mathbf{K}^{*}\left(\mathbf{L}^{-1}\right)^{3} \mathbf{U}^{2} \mathbf{C}_{i} .
\end{aligned}
$$

Using Eqn (18) and letting $\mathbf{A}^{x}=\mathbf{C}^{\mathrm{T}} \mathbf{U} \mathbf{L}^{-1}$, $\mathbf{A}^{y}=\mathbf{C}^{\mathrm{T}} \mathbf{V} \mathbf{L}^{-1}, \quad \mathbf{A}^{z}=\mathbf{C}^{\mathrm{T}} \mathbf{W} \mathbf{L}^{-1}$, Eqn (9) can be rewritten as:

$$
\left\{\begin{array}{l}
\frac{\partial \mathbf{f}^{x}}{\partial \mathbf{x}}=\mathbf{C}^{\mathrm{T}} \mathbf{K}^{*}\left(\mathbf{L}^{-1}\right)^{3} \mathbf{U}^{2} \mathbf{C}+\mathbf{E}=\mathbf{A}^{x} \mathbf{K}^{*} \mathbf{L}^{-1}\left(\mathbf{A}^{x}\right)^{\mathrm{T}}+\mathbf{E}, \\
\frac{\partial \mathbf{f}^{\mathrm{y}}}{\partial \mathbf{x}}=\mathbf{C}^{\mathrm{T}} \mathbf{K}^{*}\left(\mathbf{L}^{-1}\right)^{3} \mathbf{U} \mathbf{V} \mathbf{C}=\mathbf{A}^{y} \mathbf{K}^{*} \mathbf{L}^{-1}\left(\mathbf{A}^{x}\right)^{\mathrm{T}}, \\
\frac{\partial \mathbf{f}^{z}}{\partial \mathbf{x}}=\mathbf{C}^{\mathrm{T}} \mathbf{K}^{*}\left(\mathbf{L}^{-1}\right)^{3} \mathbf{U} \mathbf{W} \mathbf{C}=\mathbf{A}^{z} \mathbf{K}^{*} \mathbf{L}^{-1}\left(\mathbf{A}^{x}\right)^{\mathrm{T}} .
\end{array}\right.
$$

Then the stiffness of the system can be obtained as:

$$
\mathbf{K}=\left(\begin{array}{ccc}
\mathbf{A}^{x} \mathbf{K}^{*} \mathbf{L}^{-1}\left(\mathbf{A}^{x}\right)^{\mathrm{T}}+\mathbf{E} & \mathbf{A}^{x} \mathbf{K}^{*} \mathbf{L}^{-1}\left(\mathbf{A}^{y}\right)^{\mathrm{T}} & \mathbf{A}^{x} \mathbf{K}^{*} \mathbf{L}^{-1}\left(\mathbf{A}^{z}\right)^{\mathrm{T}} \\
\mathbf{A}^{y} \mathbf{K}^{*} \mathbf{L}^{-1}\left(\mathbf{A}^{x}\right)^{\mathrm{T}} & \mathbf{A}^{y} \mathbf{K}^{*} \mathbf{L}^{-1}\left(\mathbf{A}^{y}\right)^{\mathrm{T}}+\mathbf{E} & \mathbf{A}^{y} \mathbf{K}^{*} \mathbf{L}^{-1}\left(\mathbf{A}^{z}\right)^{\mathrm{T}} \\
\mathbf{A}^{z} \mathbf{K}^{*} \mathbf{L}^{-1}\left(\mathbf{A}^{x}\right)^{\mathrm{T}} & \mathbf{A}^{z} \mathbf{K}^{*} \mathbf{L}^{-1}\left(\mathbf{A}^{y}\right)^{\mathrm{T}} & \mathbf{A}^{z} \mathbf{K}^{*} \mathbf{L}^{-1}\left(\mathbf{A}^{z}\right)^{\mathrm{T}}+\mathbf{E}
\end{array}\right)
$$

The elements in the second and third columns of the tangent stiffness matrix in Eqn (8) can also be obtained in a similar way to Eqns (8) and (9).

Let $\mathbf{I}\left(\in \mathrm{R}^{3 \times 3}\right)$ denote an identity matrix. By letting $\mathbf{A}=\left(\mathbf{A}^{x}, \mathbf{A}^{y}, \mathbf{A}^{x}\right)$, the tangent stiffness matrix $\mathbf{K}$ given in Eqn (20) can be written as the sum of the linear stiffness matrix $\mathbf{K}_{E}$ and the geometrical stiffness matrix $\mathbf{K}_{G}$ as follows:

$$
\mathbf{K}=\mathbf{A} \mathbf{K}^{*} \mathbf{L}^{-1} \mathbf{A}^{\mathrm{T}}+\mathbf{I} \otimes \mathbf{E}=\mathbf{K}_{E}+\mathbf{K}_{G},
$$

where $\otimes$ denotes tensor product.

The tangent stiffness matrix presented above can be used for any pin-jointed structure in the field of elastic systems with small strain, since we have not used any further assumptions.

\section{Element importance}

In this section, we present two indexes for evaluation of element importance of a space truss, and use them in two different scenarios: to examine global effects of the indexes excluding any specific load, and to examine "local" effects of the indexes while the structure is subjected to given external loads.

\subsection{Structures without loads (first index)}

To evaluate the importance of an element, it might be straightforward to observe the change in structural perfor- mance of the structure in the two states: the intact state as well as the damaged state when the element is removed from the structure. Based on this concept, the element importance index $\alpha_{i}$ of the $i^{\text {th }}$ structural element is generally defined as follows (Pandey, Barai 1997; Gharaibeh et al. 2002):

$$
\alpha_{i}=\frac{\gamma_{0}-\gamma_{i}}{\gamma_{0}}
$$

where $\gamma_{0}$ is the structural performance index of the intact structure and $\gamma_{i}$ is the structural performance index after the failure of $i^{\text {th }}$ element.

It has been well known in structural engineering that the stiffness matrix contains detailed information of the structure, not only on its stiffness but also on its stability. Since structural performance of a structure is directly linked to its stiffness matrix, it is reasonable to evaluate the structural performance based on the tangent stiffness matrix.

Moreover, Zhang and Ohsaki (2015) stated that stability and stiffness of a structure can be characterized or indexed by the smallest eigenvalue $\lambda_{1}$ of the tangent stiffness matrix, while the zero eigenvalues corresponding to rigid-body motions have been excluded. When $\lambda_{1}$ is positive, the structure is stable; when it is negative, then the structure is unstable. Furthermore, stiffness of the structure against external loads in its weakest direction can be evaluated by the magnitude of $\lambda_{1}$. Larger $\lambda_{1}$ corresponds to higher stiffness of the structure. Hence, the smallest eigenvalue $\lambda_{1}$ is used as the first structural performance index $\gamma$ as in Eqn (22) for evaluation of element importance.

On the other hand, the determinant of the stiffness matrix, $\operatorname{det}(\mathbf{K})=\lambda_{1} \times \lambda_{2} \times \ldots \times \lambda_{3 n}$, is a theoretical tool to quantify the degree of safety of a structural system for a given performance objective (Nafday 2008). Therefore, the determinant of the tangent stiffness matrix is used as the second structural performance index $\gamma$ as in Eqn (22) for evaluation of the element importance.

A well-known static indeterminate planar truss, as shown in Figure 1, is studied to compare the element importance by using these two indexes. The structure is composed of four nodes and six bars (elements or members). The element area stiffness of all bars, $e a$, is assumed to be 1.0. The lengths of bars 1,2, 5 and 6 are 1.0. Nodes 1 and 2 are free nodes, and nodes 3 and 4 are fixed nodes. The connectivity matrix of the structure is given in Table 1.

The four smallest eigenvalues of the tangent stiffness and the results of element importance indexes are listed in Table 2. The last row of Table 2 shows the results according to the element importance index presented in C. M. Liu and X. L. Liu (2005); this index was proved by the theory of minimum of potential energy.

All of the three importance indexes result in 0.0 for bar 1 . This is because the two ends of bar 1 are constrained by the fixed nodes, such that it does not contrib- 
ute to global stiffness of the structure. That is to say, the structural stiffness is not affected after the loss of bar 1 .

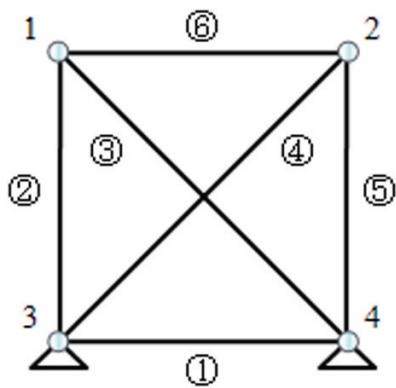

Fig. 1. Planar truss

Table 1. Connectivity matrix of the planar truss as shown in Figure 1

\begin{tabular}{ccccc}
\hline $\mathbf{C}^{s}$ & Node1 & Node2 & Node3 & Node4 \\
\hline Bar1 & 0 & 0 & 1 & -1 \\
Bar2 & 1 & 0 & -1 & 0 \\
Bar3 & 1 & 0 & 0 & -1 \\
Bar4 & 0 & 1 & -1 & 0 \\
Bar5 & 0 & 1 & 0 & -1 \\
Bar6 & 1 & -1 & 0 & 0 \\
\hline
\end{tabular}

The results by using the second proposed element importance index, based on the determinant of stiffness matrix, are almost the same as the results according to the reference by Liu and Liu, 2005. The order of element importance, from higher to lower, is $2,5,6 \rightarrow 3,4 \rightarrow 1$.

However, for the first proposed element importance index, based on the smallest eigenvalue of the stiffness matrix, the order of element importance is $2,5 \rightarrow 3,4 \rightarrow 1$, 6 , since the importance index of bar 6 is also zero. It is clear that this is incorrect, because bar 6 is important when a horizontal load is applied on the free nodes $1 \mathrm{and} /$ or 2. The reason comes from the fact that the smallest eigenvalue of the stiffness matrix is not varied after the loss of bar 6 . However, the second and fourth smallest eigenvalues of the stiffness matrix greatly decrease.
Based on the above-mentioned observations, it is found that the second element importance index based on the determinant of stiffness matrix is superior to the first one based on the smallest eigenvalue. This is because the determinant of stiffness matrix contains all the eigenvalues of the stiffness matrix, including the smallest one. In the following examples, only the second element importance index based on the determinant of stiffness matrix is used.

\subsection{Effects of support conditions}

The support conditions of the planar truss in Figure 1 are revised in this subsection to investigate the influence of support conditions on the element importance. In Figure 2 , the horizontal constraint of node 4 is released, and it is only vertically supported.

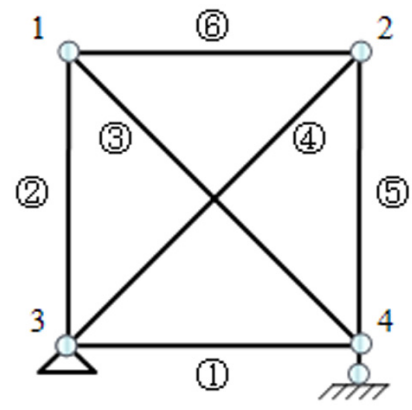

Fig. 2. Planar truss with different support conditions

The element importance indexes, based on determinant of the stiffness matrix, of all bars are summarized in Table 3 . The order of element importance is $2,5,6,1 \rightarrow 3$, 4. The element importance of bar 1 varies significantly from 0.0 to 0.8964 . Moreover, the element importance indexes of other bars also increase slightly. This is because that the degree of static indeterminacy decreases when the horizontal constraint of node 4 is released. The robustness of the system decreases and the possibility of progressive collapse increases after the loss of any element.

Table 2. Element importance result of the planar truss

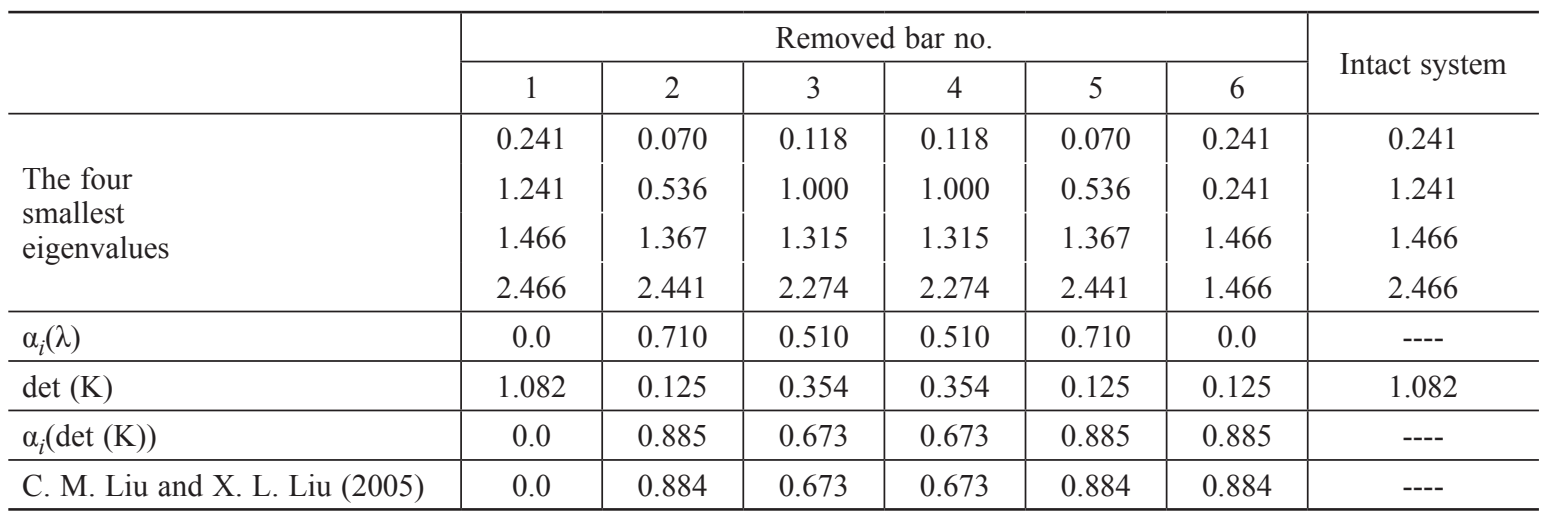


Table 3. Results of planar trusses with different support conditions as in Figure 2

\begin{tabular}{l|c|c|c|c|c|c}
\hline $\begin{array}{c}\text { Bar } \\
\text { number }\end{array}$ & 1 & 2 & 3 & 4 & 5 & 6 \\
\hline $\operatorname{det}(\mathrm{K})$ & 625.0 & 625.0 & 1767.8 & 1767.8 & 625.0 & 625.0 \\
$\alpha_{i}$ & 0.8964 & 0.8964 & 0.7071 & 0.7071 & 0.8964 & 0.8964 \\
\hline
\end{tabular}

\subsection{Effects of bar stiffness}

The area stiffness of bar 2 is increased as shown in Figure 3 (a) and the area stiffness of bars 2 and 5 is increased as shown in Figure 3(b) to study the influence of bar stiffness on the element importance. Results for all bars are given in Table 4.

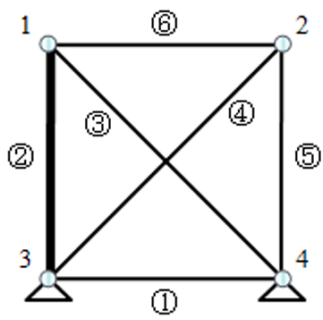

(a) Bar 2 is stiffened

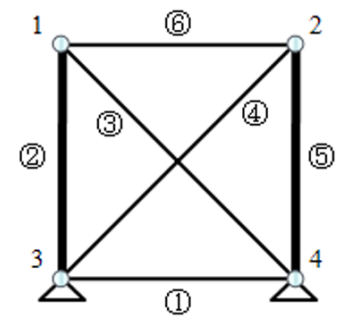

(b) Bars 2 and 5 are stiffnened
Fig. 3. Planar truss structures with different bar stiffness

Table 4. Results of planar trusses with different bar stiffness as in Figure 3

\begin{tabular}{l|c|c|c|c|c}
\hline \multicolumn{1}{c|}{$\alpha_{i}$} & 2 & 3 & 4 & 5 & 6 \\
\hline $\begin{array}{l}\text { Initial } \\
\text { structure }\end{array}$ & 0.8845 & 0.6732 & 0.6732 & 0.8845 & 0.8845 \\
\hline$e a_{2}=1.5$ & 0.9199 & 0.6602 & 0.6602 & 0.8799 & 0.8799 \\
\hline$e a_{2}=2$ & 0.9387 & 0.6532 & 0.6532 & 0.8774 & 0.8774 \\
\hline $\begin{array}{l}e a_{2}=e a_{5}= \\
1.5\end{array}$ & 0.9166 & 0.6460 & 0.6460 & 0.9166 & 0.8748 \\
\hline
\end{tabular}

It can be seen from Table 4 that the element importance index of bar 2 increases, when its bar stiffness is increased. Meanwhile, the element importance indexes of other bars reduce. In the case that the area stiffness of bars 2 and 5 is both increased, the element importance indexes of both bars increase. It can be concluded that the element importance index increases with the increase of bar stiffness. Intuitionally the bars with higher stiffness sustain higher external loads in the load path, and therefore, it becomes more important to the structure.

\subsection{Structures under loads (second index)}

The element importance index considering the load effect is studied in this subsection. In this situation, the element importance index is revised as follows:

$$
\alpha_{i}=\frac{\gamma_{0}-\gamma_{i}^{f}}{\gamma_{0}},
$$

where $\gamma_{i}^{f}$ is the structural performance index after the failure of $i^{\text {th }}$ element under the external load.
In general, the stiffness of a space structure is not large, thus, its deformation under external loads maybe significant, which leads to a large geometry variation; Moreover, the geometrical stiffness of the structure will change significantly, according to the theory in Section 1.3. Accordingly, the geometric nonlinearity should be considered in the evaluation of element importance index.

Figure 4 illustrates the geometric nonlinearity effect on the structure. $\mathrm{K}^{0}$ refers the initial tangent stiffness of the structure. Under the external load vector $\mathrm{P}$, the initial estimation of displacement can be obtained as $\mathrm{U}^{1}$ as shown in the figure by applying a linear analysis. However, the system is unbalanced at this moment, and the unbalanced force $\mathrm{R}^{1}$ can be calculated at the displacement $\mathrm{U}^{1}$. Then the new tangent stiffness of the structure $\mathrm{K}^{1}$ will be obtained with the new geometry $\mathrm{X}^{1}=\mathrm{X}^{0}+\mathrm{U}^{1}$. The new displacement $\mathrm{U}^{2}$ can be obtained with a linear analysis when the unbalanced force $\mathrm{R}^{1}$ is applied in the opposite direction, which will leads to a new unbalanced force $\mathrm{R}^{2}$. Repeat the previous steps until the unbalanced force vector is small enough.

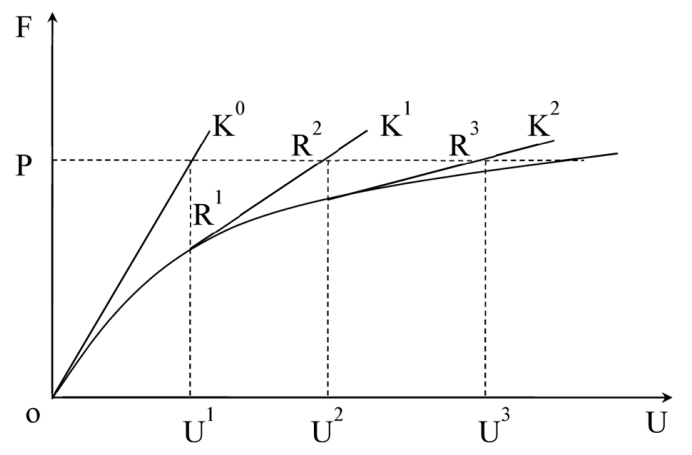

Fig. 4. Effects of geometric nonlinearity

Two different loading patterns of the planar trusses in Figure 1 are considered as shown in Figure 5: in one pattern it is under a horizontal load, and in the other pattern it is under a vertical load, both are applied at the free node 1 . The element importance indexes of all members under different load levels are summarized in Tables 5 and 6. It should be noted that the load levels in these tables are the ratios of external loads to the bar area stiffness.

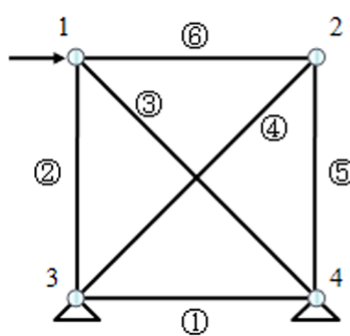

(a) Horizontal load

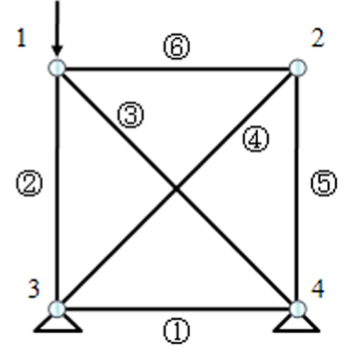

(b) Vertical load
Fig. 5. Planar truss under different loading patterns 
Table 5. Element importance indexes of the planar truss under horizontal load as shown in Figure 5(a)

\begin{tabular}{c|c|c|c|c|c}
\hline \multirow{2}{*}{$\begin{array}{c}\text { Load } \\
\text { level }\end{array}$} & \multicolumn{5}{|c}{$\alpha_{i}$} \\
\cline { 2 - 6 } & Bar 2 & Bar 3 & Bar 4 & Bar 5 & Bar 6 \\
\hline 0 & 0.8845 & 0.6732 & 0.6732 & 0.8845 & 0.8845 \\
0.001 & 0.8863 & 0.6750 & 0.6722 & 0.8839 & 0.8841 \\
0.003 & 0.8866 & 0.6782 & 0.6700 & 0.8825 & 0.8833 \\
0.005 & 0.8884 & 0.6788 & 0.6693 & 0.8823 & 0.8830 \\
0.007 & 0.8906 & 0.6815 & 0.6681 & 0.8818 & 0.8824 \\
0.009 & 0.8902 & 0.6845 & 0.6672 & 0.8815 & 0.8819 \\
\hline
\end{tabular}

Table 6. Element importance indexes of the planar truss under horizontal load as shown in Figure 5(b)

\begin{tabular}{c|c|c|c|c|c}
\hline \multirow{2}{*}{$\begin{array}{c}\text { Load } \\
\text { level }\end{array}$} & \multicolumn{5}{|c}{$\alpha_{i}$} \\
\cline { 2 - 6 } & Bar 2 & Bar 3 & Bar 4 & Bar 5 & Bar 6 \\
\hline 0 & 0.8845 & 0.6732 & 0.6732 & 0.8845 & 0.8845 \\
0.001 & 0.8872 & 0.6736 & 0.6733 & 0.8821 & 0.8846 \\
0.003 & 0.8950 & 0.6767 & 0.6739 & 0.8599 & 0.8854 \\
0.005 & 0.8983 & 0.6771 & 0.6751 & 0.8464 & 0.8858 \\
0.007 & 0.9047 & 0.6820 & 0.6748 & 0.8372 & 0.8873 \\
0.009 & 0.9130 & 0.6883 & 0.6756 & 0.8255 & 0.8896 \\
\hline
\end{tabular}

It can be seen from these tables that the element importance indexes vary with different load levels. For bar 3, the element importance index increases with the increase of load level, whereas the element importance index of bar 5 reduces with the increase of load level.

Furthermore, the trend of variation of element importance index might be affected by the loading patterns. For example, the element importance index of a bar increases with the reduction of load level under the horizontal load; on the contrary, it increases with the increase of load level under the vertical load. Therefore, the order of element importance is changed to be $2 \rightarrow 6 \rightarrow 5 \rightarrow 3 \rightarrow 4$.

Moreover, the element importance indexes under zero loading in Tables 5 and 6 are the same as those in Table 2. This shows that the element importance indexes proposed in Eqns (22) and (23) are consistent when the external loads are not considered. In other words, Eqn (22) is a special case of Eqn (23). From the above analysis, it can be concluded that the element importance index may be influenced by the load level as well as loading pattern.

\subsection{First-order infinite mechanism}

A three-bar pin-jointed structure shown in Figure 6 is studied in this subsection. The element area stiffness of all bars, $e a$, is assumed to be 1.0. The lengths of all bars are 1.0. There is a vertical load, $\mathrm{P}$, applied at node 4 in vertical direction. The element importance indexes are summarized in Table 7.

Table 7. Results of a three-bar pin-jointed structure

\begin{tabular}{c|c|c|c}
\hline \multirow{2}{*}{$\begin{array}{c}\text { Load } \\
\text { values }\end{array}$} & \multicolumn{3}{|c}{$\alpha_{i}$} \\
\cline { 2 - 4 } & Bar 1 & Bar2 & Bar3 \\
\hline 0 & 0.5000 & 0.5000 & 1.0000 \\
$1.0 \times 10^{-7}$ & 0.5000 & 0.5000 & 0.9997 \\
$1.0 \times 10^{-6}$ & 0.5000 & 0.5000 & 0.9996 \\
$1.5 \times 10^{-6}$ & 0.5000 & 0.5000 & 0.9995 \\
$3.0 \times 10^{-6}$ & 0.5000 & 0.5000 & 0.9994 \\
\hline
\end{tabular}

It can be seen from Table 7 that the element importance index of bar 3 is 1.0 without external loads. This is because the structure becomes a mechanism after the loss of bar 3. However, the element importance index of bar 3 decreases when the load level increases. The reason of this is that the first-order mechanism disappears when bars 1 and 2 do not lie on the same line, and therefore, they provide stiffness to the structure in the vertical direction. Accordingly, the method proposed in this paper can also be applied to the structures consisting of the firstorder infinite mechanisms.

\section{Element importance of space trusses}

\subsection{A square pyramid space truss}

A square pyramid space grid, as shown in Figure 7, is chosen as an example to study the element importance of complicated space trusses. The grid number of this system is $8 \times 8$, and the length of the grid is $2.5 \mathrm{~m}$. The height of the system is $1.5 \mathrm{~m}$. The loads are applied at the upper nodes in the vertical direction. The upper surrounding nodes are assumed to be fixed. The members are made in steel and their elastic modulus is $2.1 \times 10^{11} \mathrm{~N} / \mathrm{m}^{2}$. The members of the upper chord and lower chord are the steel pipes with $60 \mathrm{~mm}$ in diameter and $4 \mathrm{~mm}$ in thickness. For the diagonal web bars, steel pipes of $51 \mathrm{~mm}$ in diameter and $4 \mathrm{~mm}$ in thickness are used.

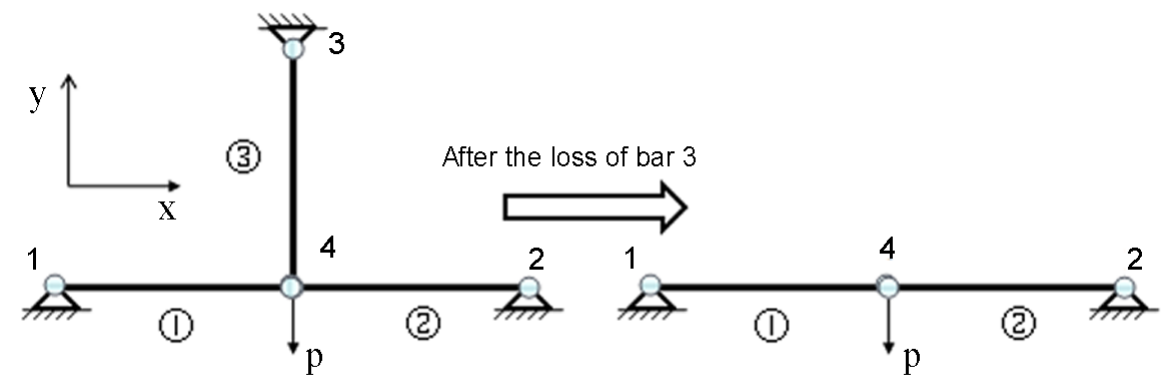

Fig. 6. A three-bar pin-jointed structure 


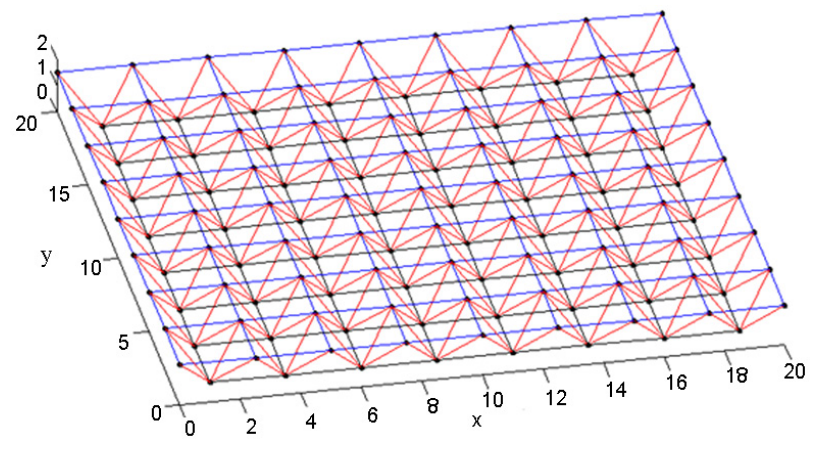

Fig. 7. A square pyramid space grid system

Before the calculation, it is reasonable to check the structural symmetry to simplify the non-linear analysis. This square pyramid space grid not only has the axial symmetry but also has the central symmetry. Then a $1 / 8$ structure was used as shown in Figure 8, where the bar numbers are also given.

The element importance indexes of the space truss under different load levels are given in Table 8. It should be noted in this table that bars 9-100 are the upper chords, bars 152-217 are the lower chords and bars 257-360 are the diagonal webs. Moreover, two ends of bars $73-76$ are restrained by fixed nodes, and their element importance indexes are 0.0. Therefore, these bars are deleted from the system. It can be observed in Table 8 that the element importance indexes of all bars are affected by the load level but this effect is minor. The element importance indexes of all bars reduce with the increase of load levels.

The element importance index of bar 166 is always the highest under different load levels. Moreover, element importance indexes of bars 159 and 152 are also very high. These bars are located at the surrounding of the lower chord plane, and they are directed perpendicularly to the support side. Among the upper chords, the element importance index of bar 36, which is located at the center
Table 8. Results of a square pyramid truss

\begin{tabular}{c|c|c|c|c}
\hline \multirow{2}{*}{$\begin{array}{c}\text { Bar } \\
\text { number }\end{array}$} & \multicolumn{4}{|c}{ Load level $(\mathrm{kN})$} \\
\cline { 2 - 5 } & 0 & 10 & 20 & 30 \\
\hline 9 & 0.6308 & 0.6301 & 0.6294 & 0.6287 \\
18 & 0.7259 & 0.7253 & 0.7248 & 0.7242 \\
27 & 0.7526 & 0.7521 & 0.7516 & 0.7510 \\
33 & 0.6506 & 0.6500 & 0.6493 & 0.6485 \\
36 & 0.7603 & 0.7597 & 0.7592 & 0.7586 \\
82 & 0.7127 & 0.7122 & 0.7116 & 0.7110 \\
84 & 0.7507 & 0.7502 & 0.7497 & 0.7492 \\
91 & 0.7510 & 0.7505 & 0.7499 & 0.7494 \\
100 & 0.7602 & 0.7596 & 0.7591 & 0.7585 \\
152 & 0.8517 & 0.8514 & 0.8511 & 0.8508 \\
159 & 0.8570 & 0.8567 & 0.8564 & 0.8561 \\
160 & 0.7979 & 0.7975 & 0.7971 & 0.7967 \\
166 & 0.8579 & 0.8576 & 0.8573 & 0.8570 \\
168 & 0.7817 & 0.7812 & 0.7808 & 0.7803 \\
201 & 0.8009 & 0.8005 & 0.8002 & 0.7998 \\
203 & 0.7214 & 0.7209 & 0.7204 & 0.7199 \\
209 & 0.7890 & 0.7886 & 0.7881 & 0.7877 \\
217 & 0.7783 & 0.7779 & 0.7774 & 0.7769 \\
257 & 0.5040 & 0.5032 & 0.5023 & 0.5014 \\
290 & 0.6892 & 0.6887 & 0.6881 & 0.6875 \\
296 & 0.7242 & 0.7238 & 0.7234 & 0.7230 \\
322 & 0.6999 & 0.6994 & 0.6989 & 0.6984 \\
328 & 0.7209 & 0.7205 & 0.7200 & 0.7196 \\
332 & 0.7190 & 0.7186 & 0.7181 & 0.7177 \\
353 & 0.5156 & 0.5147 & 0.5138 & 0.5128 \\
360 & 0.7189 & 0.7185 & 0.7181 & 0.7176 \\
\hline & & & & \\
\hline
\end{tabular}

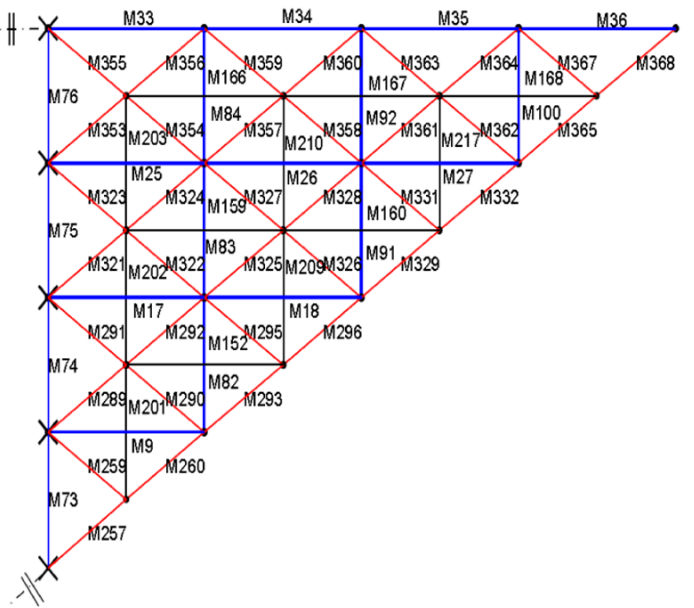

(b) Planar images

Fig. 8 . The $1 / 8$ structure system 
of the upper chord plane, is the highest. Bar 296 is the most important among the diagonal webs. It can also be concluded from Table 8 that the lower chords are more important than the upper chords and the diagonal webs. When the distance between upper chords and the support side is farther, their importance indexes larger.

\subsection{Effects of load distributions}

In practice, the load of a space truss is very complex. In this subsection, four loading patterns are given as shown in Figure 9 to investigate the effects of load patterns on the element importance index: Loading pattern $\mathrm{I}$ is a vertical symmetric uniform load, Loading patter II is a vertical anti-symmetric load, Loading pattern III is the vertical half-span load, and Loading pattern IV is a horizontal uniform load. The load level applied at every node for the first three loading patterns is $20.0 \mathrm{kN}$, and the nodal load level is $5.0 \mathrm{kN}$ for the fourth horizontal loading pattern.

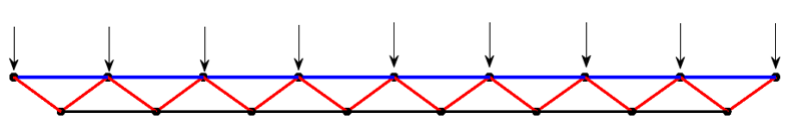

(a) I: Vertical symmetric loads

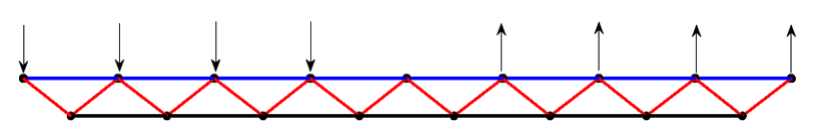

(b) II: Vertical anti-symmetric loads

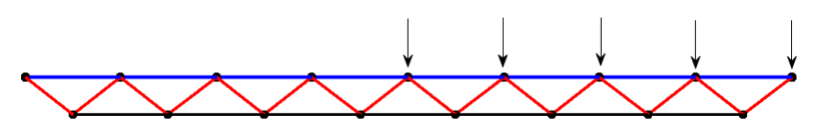

(c) III: Vertical half-span loads

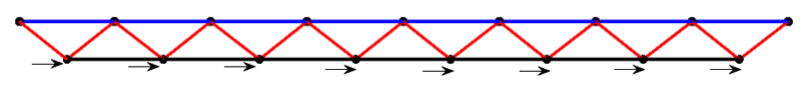

(d) IV: Horizontal uniform loads

Fig. 9. Loading patterns

Table 9. Results under different loading patterns

\begin{tabular}{c|c|c|c|c}
\hline $\begin{array}{c}\text { Bar } \\
\text { number }\end{array}$ & $\begin{array}{c}\text { Loading } \\
\text { pattern I }\end{array}$ & $\begin{array}{c}\text { Loading } \\
\text { pattern II }\end{array}$ & $\begin{array}{c}\text { Loading } \\
\text { pattern III }\end{array}$ & $\begin{array}{c}\text { Loading } \\
\text { pattern IV }\end{array}$ \\
\hline 9 & 0.6308 & 0.6300 & 0.6308 & 0.6308 \\
18 & 0.7258 & 0.7252 & 0.7259 & 0.7259 \\
36 & 0.7602 & 0.7596 & 0.7603 & 0.7603 \\
100 & 0.7601 & 0.7595 & 0.7602 & 0.7602 \\
160 & 0.7980 & 0.7974 & 0.7979 & 0.7979 \\
166 & 0.8579 & 0.8575 & 0.8579 & 0.8579 \\
201 & 0.8009 & 0.8004 & 0.8009 & 0.8009 \\
257 & 0.5041 & 0.5029 & 0.5041 & 0.5040 \\
296 & 0.7243 & 0.7236 & 0.7242 & 0.7242 \\
360 & 0.7190 & 0.7184 & 0.7189 & 0.7190 \\
\hline
\end{tabular}

Table 9 shows the element importance indexes of some selected bars under different loading patterns. It can be found that the element importance indexes are different when the structure is subjected to different load patterns. However, this effect is minor and the order of element importance is not changed. For all loading patterns, the importance index of bar 166 is always the highest. Among the upper chords, the element importance index of bar 36 is the highest. Bar 296 is the most important among the diagonal webs.

It can be concluded from the above-mentioned discussions that the order of element importance is not changed under general external loads. The influence of load levels and loading patterns on the element importance is not significant. Therefore, for simplicity, the element importance can be estimated without considering the external loads for the square pyramid truss studied in this subsection.

\section{Conclusions}

Based on the consensus that elements transferring more loads in the load path are generally more important in the structural, the element importance index is defined as the change in structural stiffness after removing the element from the system. The smallest eigenvalue and the determinant of tangent stiffness matrix are chosen as the structural performance indexes.

A well-known truss structure was studied to compare these two indexes. The results show that the index based on determinant of tangent stiffness matrix is superior to that based on the smallest eigenvalue. The effects of support conditions, bar area stiffness and external loads on the proposed element importance index are then investigated using the planar truss structure.

Finally, a square pyramid space truss is selected as an illustrative example. It is found that the lower chords are more important than the upper chords as well as the diagonal webs. When the distance between the upper chords and the support side is farther, the importance index becomes larger. Moreover, the element importance of this space truss is primarily related to the linear stiffness matrix. Therefore, the element importance index without load effect can be used to approximately evaluate the element importance of the square pyramid space grids.

\section{Acknowledgements}

The work presented in this article was supported by the National Natural Science Foundation of China (Grant No. 51308106, No. 51578133), a Project Funded by the Priority Academic Program Development of Jiangsu Higher Education Institutions, and the Excellent Young Teachers Program of Southeast University. Authors also thank the anonymous reviewers for their valuable comments and thoughtful suggestions which improved the quality of the presented work. 


\section{References}

Affan, A.1987. Collapse of double-layer space grids: $\mathrm{PhD}$ thesis. Cambridge University, Cambridge, U.K.

Affan, A.; Calladine, C. R.1996. Initial bar tensions in pin-jointed assemblies, International Journal of Space Structures 4(1): 1-16.

Blandford, G. E. 1996. Progressive failure analysis of inelastic space truss structures, Computers \& Structures 58(5): 981-990. https://doi.org/10.1016/0045-7949(95)00217-5

Cai, J. G.; Feng, J. 2015. Form-finding of tensegrity structures using an optimization method, Engineering Structures 104: 126-132. https://doi.org/10.1016/j.engstruct.2015.09.028

De Biagi, V. 2016. Structural behavior of a metallic truss under progressive damage, International Journal of Solids and Structures 82: 56-64.

http://dx.doi.org/10.1016/j.ijsolstr.2015.12.016

Djamaluddin, F.; Abdullah, S.; Ariffin, A. K.; Nopiah, Z. M. 2015. Non-linear finite element analysis of bitubal circular tubes for progressive and bending collapses, International Journal of Mechanical Sciences 99: 228-236. https://doi.org/10.1016/j.ijmecsci.2015.06.001

Ellingwood, B. R. 2005. Building design for abnormal loads and progressive collapse, Computer Aided Civil Infrastructure Engineering 20(3): 194-205. https://doi.org/10.1111/j.1467-8667.2005.00387.x

Ei-Sheikh, A. 1997. Sensitivity of space trusses to sudden member loss, International Journal of Space Structures 12(1): $31-41$.

Guo, L. H.; Gao, S.; Fu, F. 2015. Structural performance of semi-rigid composite frame under column loss, Engineering Structures 95: 112-126.

https://doi.org/10.1016/j.engstruct.2015.03.049

Gharaibeh, E. S.; Frangopol, D. M.; Onoufriou, T. 2002. Reliability-based importance assessment of structural members with applications to complex structures, Computers \& Structures 80(12): 1113-1131.

https://doi.org/10.1016/S0045-7949(02)00070-6

Jiang, X. F.; Chen, Y. Y. 2012. Progressive collapse analysis and safety assessment method for steel truss roof, Journal of Performance of Constructed Facilities 26(3): 230-240. https://doi.org/10.1061/(ASCE)CF.1943-5509.0000236

Kim, J.; Park, J. 2014. Design of special truss moment frames considering progressive collapse, International Journal of Steel Structures 14(2): 331-343. https://doi.org/10.1007/s13296-014-2013-1

Liu, C. M.; Liu, X. L. 2005. Stiffness-based evaluation of component importance and its relationship with redundancy, Journal of Shanghai Jiaotong University 39(5): 746-750 (in Chinese).

Malla, R. B.; Nalluri, N. 1995. Dynamic effects of member failure on the response of truss type space structures, Journal of Spacecraft and Rockets 32(3): 545-551. https://doi.org/10.2514/3.26649

Malla, R. B.; Serrette, R. L. 1996. Double-layer grids: review of dynamic analysis methods and special topics, Journal of Structural Engineering ASCE 122(8): 882-892.

https://doi.org/10.1061/(ASCE)0733-9445(1996)122:8(882)

Malla, R. B.; Agarwal, P.; Ahmad, R. 2011. Dynamic analysis methodology for progressive failure of truss structures considering inelastic postbuckling cyclic member behaviour, Engineering Structures 33(5): 1503-1513. https://doi.org/10.1016/j.engstruct.2011.01.022

Mashhadiali, N.; Kheyroddin, A. 2014. Progressive collapse assessment of new hexagrid structural system for tall buildings, The Structural Design of Tall and Special Buildings 23(12): 947-961. https://doi.org/10.1002/tal.1097
Milana, G.; Olmati, P.; Gkoumas, K.; Bontempi, F. 2015. Ultimate capacity of diagrid systems for tall buildings in the nominal configuration and the damaged state, Periodica Polytechnica Civil Engineering 59(3): 381-391. https://doi.org/10.3311/PPci.7795

Ministry of Housing and Local Government. 1968. Collapse of flats at Ronan point, Canning Town. London: HMSO, $46-51$.

Morris, N. F.1993. Effect of member snap on space truss collapse, Journal of Engineering Mechanics ASCE 119(4): 870-886. https://doi.org/10.1061/(ASCE)07339399(1993)119:4(870)

Murtha-Smith, E. 1988. Alternate path analysis of space trusses for progressive collapse, Journal of Structural Engineering ASCE 114(9): 1978-1999. https://doi.org/10.1061/ (ASCE)0733-9445(1988)114:9(1978)

Nafday, A. M. 2008. System safety performance metrics for skeletal structures, Journal of Structural Engineering ASCE 134(3): 499-504. https://doi.org/10.1061/ (ASCE)0733-9445(2008)134:3(499)

Nafday, A. M. 2011. Consequence-based structural design approach for black swan events, Structural Safety 33(1): 108-114. https://doi.org/10.1016/j.strusafe.2010.09.003

Olmati, P.; Gkoumas, K.; Brando, F.; Cao, L. L. 2013. Consequence-based robustness assessment of a steel truss bridge, Steel and Composite Structures 14(4): 379-395. https://doi.org/10.12989/scs.2013.14.4.379

Ohsaki, M.; Zhang, J. Y. 2006. Stability conditions of prestressed pin-jointed structures, International Journal of Nonlinear Mechanics 41(10): 1109-1117. https://doi.org/10.1016/j.ijnonlinmec.2006.10.009

Pandey, P. C.; Barai, S. V. 1997. Structural sensitivity as a measure of redundancy, Journal of Structural Engineering ASCE 123(3): 360-364. https://doi.org/10.1061/ (ASCE)0733-9445(1997)123:3(360)

Salem, H. M.; Helmy, H. M. 2014. Numerical investigation of collapse of the Minnesota I-35W bridge, Engineering Structures 59: 635-645. https://doi.org/10.1016/j.engstruct.2013.11.022

Sebastian, W. M. 2004. Collapse considerations and electrical analogies for statically indeterminate structures, Journal of Structural Engineering 130(10): 1445-1453. https://doi. org/10.1061/(ASCE)0733-9445(2004)130:10(1445)

Sheidaii, M. R.; Parke, G. A. R.; Abedi, K.; Behravesh, A. 2001. Dynamic Snap-through buckling of truss-type structures, International Journal of Space Structures 16(2):85-93. https://doi.org/10.1260/0266351011495197

Stylianidis, P. M.; Nethercot, D. A. 2015. Modelling of connection behaviour for progressive collapse analysis, Journal of Constructional Steel Research 113: 169-184. https://doi.org/10.1016/j.jcsr.2015.06.008

U.S. Dept. of Defense (DoD). 2009. Design of buildings to resist progressive collapse, UFC 4-023-03. Washington, DC, USA.

U.S. General Services Administration (GSA). 2003. Progressive collapse analysis and design guidelines for new federal office buildings and major modernization projects. Washington, DC, USA.

Wang, B. 1994. Linear dynamic response of truss structures during sudden member failure: Ms thesis. Department of Civil Engineering, University of Connecticut, Storrs.

Wu, X.; Blockley, D. I.; Woodman, N. J. 1993a. Vulnerability analysis of structural systems. I: Rings and clusters, Civil Engineering Systems 10(4): 301-317. https://doi.org/10.1080/02630259308970130

Wu, X.; Blockley, D. I.; Woodman, N. J. 1993b. Vulnerability analysis of structural systems. II: Failure scenarios, Civil Engineering Systems 10(4): 319-333. https://doi.org/10.1080/02630259308970131 
Zhang, J. Y.; Ohsaki, M. 2006. Adaptive force density method for form-finding problem of tensegrity structures, International Journal of Space Structures 43(18/19): 5658-5673. https://doi.org/10.1016/j.ijsolstr.2005.10.011
Zhang, J. Y.; Ohsaki, M. 2015. Tensegrity structures: form, stability, and symmetry. Springer.

https://doi.org/10.1007/978-4-431-54813-3

Jianguo CAI. He is an associate Professor in Key Laboratory of Concrete and Prestressed Concrete Structure of Ministry of Education at Southeast University, Nanjing, China. He is also working for the National Prestress Engineering Research Center of Southeast University. He is the author of about 80 papers, published in both national and international conference proceedings and journals. His research interests focus on Long-span Structures and Deployable Structures.

Wenwen JIA. She is a graduate student in School of Civil Engineering at Southeast University, Nanjing, China. Her research interests focus on Prestressed Structures.

Jian FENG. He is currently working as a Professor in Key Laboratory of Concrete and Prestressed Concrete Structure of Ministry of Education, Southeast University, Nanjing, China. He is also working for the National Prestress Engineering Research Center of Southeast University. He is the author of about 150 papers, published in both national and international conference proceedings and journals. His research interests focus on Structural Design, Prestressed Structures and Precast Structures.

Fang WANG. He is a master student in Key Laboratory of Concrete and Prestressed Concrete Structure of Ministry of Education at Southeast University, Nanjing, China.

Yixiang XU. He is a Lecturer in the Departments of Civil Engineering at the University of Strathclyde, Glasgow, UK. He is the author of a number of papers, published in conference proceedings and journals. He is interested in the area of numerical study of advanced space structures as well as and design and analysis of infrustructural structutres, e.g. portal frame and web crippling. 